\title{
Advances in Elemental Spectroscopy Logging: A Cased Hole Application Offshore West Africa
}

\author{
Irewole Ayodele ${ }^{1}$, Chiara Cavalleri ${ }^{2}$, Adeleke Orimolade $^{1} \&$ Babafemi Falaye $^{1}$ \\ ${ }^{1}$ South Atlantic Petroleum (SAPETRO), Lagos, Nigeria \\ ${ }^{2}$ Schlumberger, Aberdeen, UK \\ Correspondence: Irewole Ayodele, Head of Geosciences, South Atlantic Petroleum (SAPETRO), Lagos, Nigeria. \\ Tel: 234-1462-3100. E-mail: irewole.ayodele@sapetro.com; irewole.ayodele@gmail.com
}

Received: October 26, 2017

Accepted: November 6, 2017

Online Published: November 30, 2017

doi:10.5539/jgg.v9n4p63

URL: http://dx.doi.org/10.5539/jgg.v9n4p63

The research is financed by (South Atlantic Petroleum Limited (SAPETRO), SAPETRO Towers, 1 Adeola Odeku, Victoria Island, Lagos, Nigeria).

\begin{abstract}
Rising costs for exploration and developments and more stringent need to secure any additional drop of oil have put operators' margins under increasing pressure. Coupled with the recent oil price decline, this call for efficiency and diligence to be the main drivers for any formation evaluation and planning for development and production.

The reservoirs in western offshore Africa are so diverse in their settings that two reservoirs hardly show any correlation. The rifting of the African plate from the South American plate has introduced significant geological complexities in characterizing rocks using petrophysical analysis for reservoir rock quality and hydrocarbon potential. These complexities include variable and heterogenous mineralogy, unresolved clay characterization, fresh formation waters with variable salinity, thin shale laminations and grain size variations contributing to low resistivity low contrast pay generation. Moreover, accessibility to the target reservoir is difficult and risky, therefore formation evaluation must be performed behind casing.

Advanced and fit-for-purpose logging technologies and computational methods are needed to surmount these complexities.

The high definition spectroscopy tool is the latest development in wireline spectroscopy measurements. Its technological advances revolutionize the neutron-induced gamma ray methodology to support robust lithology and saturation interpretation in formations with complex mineralogy and fluid content. The ability to determine both the matrix mineral composition and total organic carbon (TOC) are instrumental to the geoscientist, petrophysicist, reservoir engineer and completion engineer.

In the region, the use of high definition spectroscopy measurement has been pioneered while pursuing better understanding of rock composition and more accurate reservoir models in complex lithology and fresh formation waters with low resistivity contrast. The results are beneficial at the various stages of a field development and provide critical input to the petrophysical reserves estimate.

In the example described in this paper, this innovative technology has proven to be critical to evaluate a complex reservoir system independent of the water salinity and resistivity offshore Gulf of Guinea, even with logging behind casing. A comprehensive set of quality outputs is made available for accurate reservoir quality; the logs data processing was performed within the critical-hours after logging to enable informed decision making.
\end{abstract}

Keywords: elemental spectroscopy, organic carbon content, saturation

\section{Introduction}

Logging plays a primary role in proper description of existing reservoir systems and screening of new productive sand units for optimal development plan and production strategy. Effective decisions need to be made on time for quick yet robust selection of completion intervals and recoverable rates.

The knowledge of rock composition and mineralogy of the formation become particularly critical to support the 
petrophysical reserves analysis and assist completion and development strategies. The latest generation of neutroninduced gamma ray technology provides these important data with applications both in open and cased holes.

This study documents the experience of alternative logging and analytical methods applied to a development well drilled through a complex shaly sand sequence offshore Gulf of Guinea. The development well drilled in a difficult sector of the field and an extended formation evaluation program was planned to be deployed through open-hole logging. However, the accessibility of the target reservoirs was risky; hence the decision to immediately set the casing after total depth was reached and complete the evaluation program with logging behind casing.

The lithology ranges from coarse sandstones and conglomerates to thin layers of silty sands and shale intercalated by limestone rock at certain levels. Oils from the main sand reservoirs within the field are heavy, low sulfur crude oil. Clay content and fresh to very fresh water salinities affect any conventional evaluation and resistivity-based method. Based on the experience gained in offset wells, the measured resistivity in water and hydrocarbon could be similar or even present reverse profile.

A first attempt was made to delineate reservoir properties and flag any producible intervals using cased-hole density and neutron porosity combined to resistivity recorded while drilling. The analysis of the data provides useful information but it clearly appears insufficient to indicate the fluid content with the required confidence. There is no clear hydrocarbon signature from the logs. However, experience of the local geology shows that the resistivity profile may be variable and finding oil where the formation resistivity is low is not uncommon.

To ensure more comprehensive data analysis, the new generation spectroscopy measurement was recorded in addition to cased hole density, neutron porosity, and sonic logs. This successfully confirmed the connate movable fluid, solving for the uncertainties related to conventional logs interpretation.

In addition to objective mineralogy and matrix properties, the high definition spectroscopy uses a novel methodology to determine the organic carbon content which is directly used to calculate the fluids volume across the reservoir of interest. The resulting quantification of the oil saturation is direct and independent of resistivitybased methods, variation in water salinity and clay content. This is the first successful application of the methodology in the region and challenging cased-hole logging conditions in West Africa.

\section{Method}

The high-definition elemental spectroscopy technology adopted for the study combines a very high-resolution scintillator and a high-output pulsed neutron generator to measure an expanded set of elements. This, in turn, increases the ability to better understand and properly interpret complex formations, adding value to any petrophysical analysis. In addition to computing elements derived from capture spectroscopy, robust inelastic yields are measured to enable a wide range of outputs. This include direct measurement of total carbon concentration, from which the amount of inorganic carbon associated with carbonate minerals is subtracted to determine the total organic carbon (TOC). The calculation was done independently and doesn't require local calibration. Hydrocarbon saturation can then be directly inferred from TOC $\log$ and a porosity log, regardless of formation water resistivity, saturation equation parameters, or clays effect. The ability to merge, capture and inelastic data significantly improve precision, accuracy, and consistent interpretation behind casing.

As shown in Figure 1, the tool uses an electrical neutron source (high-output Pulse Neutron Generator) coupled to a large cerium-doped lanthanum bromite $(\mathrm{LaBr} 3: \mathrm{Ce})$ gamma ray detector with high-temperature photomultiplier. The very fast scintillator provides high light outputs and excellent spectral resolution for robust measurements and enhanced statistical precision in a wide range of environments.

To maximize the benefit and use of the state-of-art transmitter-detector combination, the spectroscopy acquisition system is optimized based on extended tool characterization in real and laboratory formations, assisted by mathematical modeling based on the industry-standard Monte Carlo N-particle transport code. 

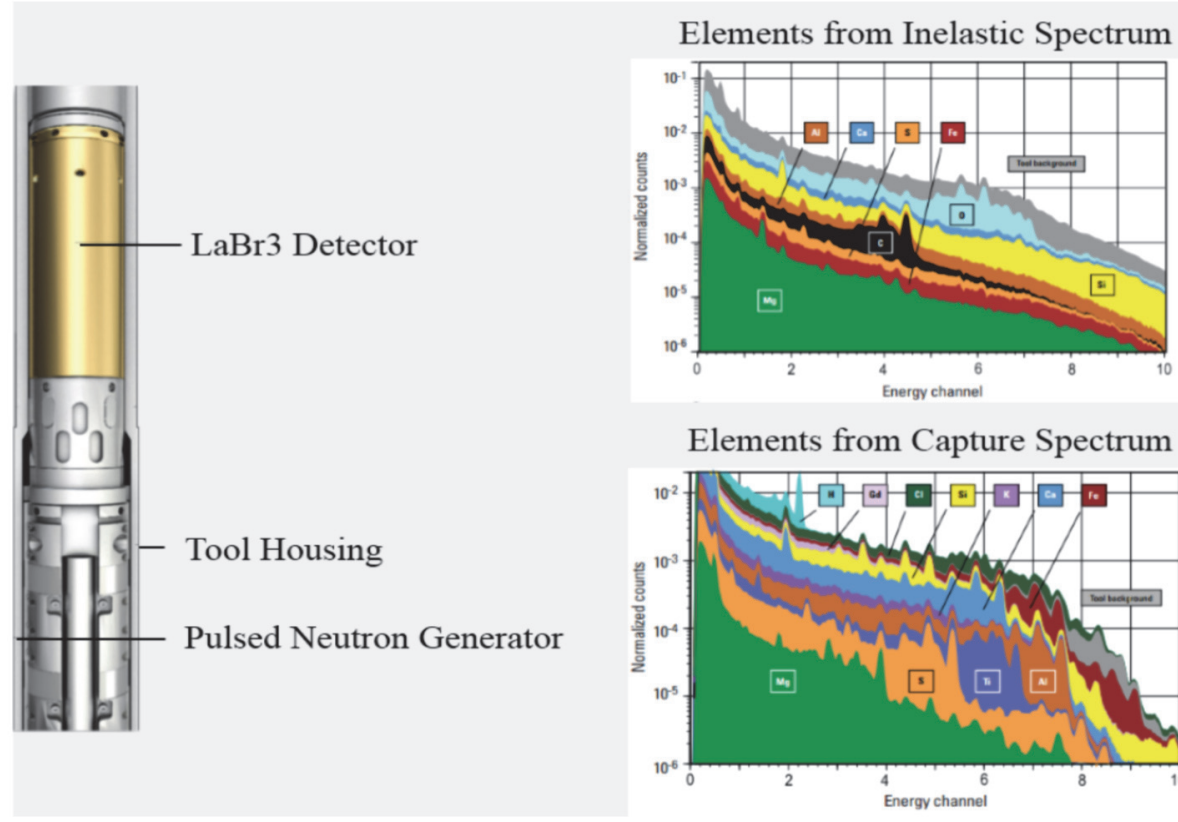

Elements from Capture Spectrum

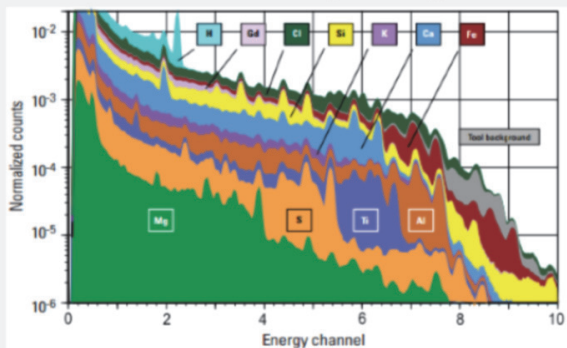

Figure 1. High definition spectroscopy - measurement section and output elements

The high definition spectroscopy service is recorded behind 7-inches casing to unlock any uncertainty related to the conventional analysis and explain the response of the other logs measurement recorded in the difficult condition.

The logging parameters and speed of the data acquisition are optimized to minimize uncertainties in the computed elemental dry-weight fractions, including the more challenging elements to be detected, such as the Carbon.

The data processing to transform the gamma ray spectra into petrophysical properties was performed in an interactive manner using an innovative interpretation platform. The main steps of the processing workflow are described in the graphic shown in Figure 2. Proper environmental corrections were applied to account for casing and cement effect. The data from the main and repeat logging pass are accurately stacked for enhanced accuracy and precision of the elements distribution and computed answers.

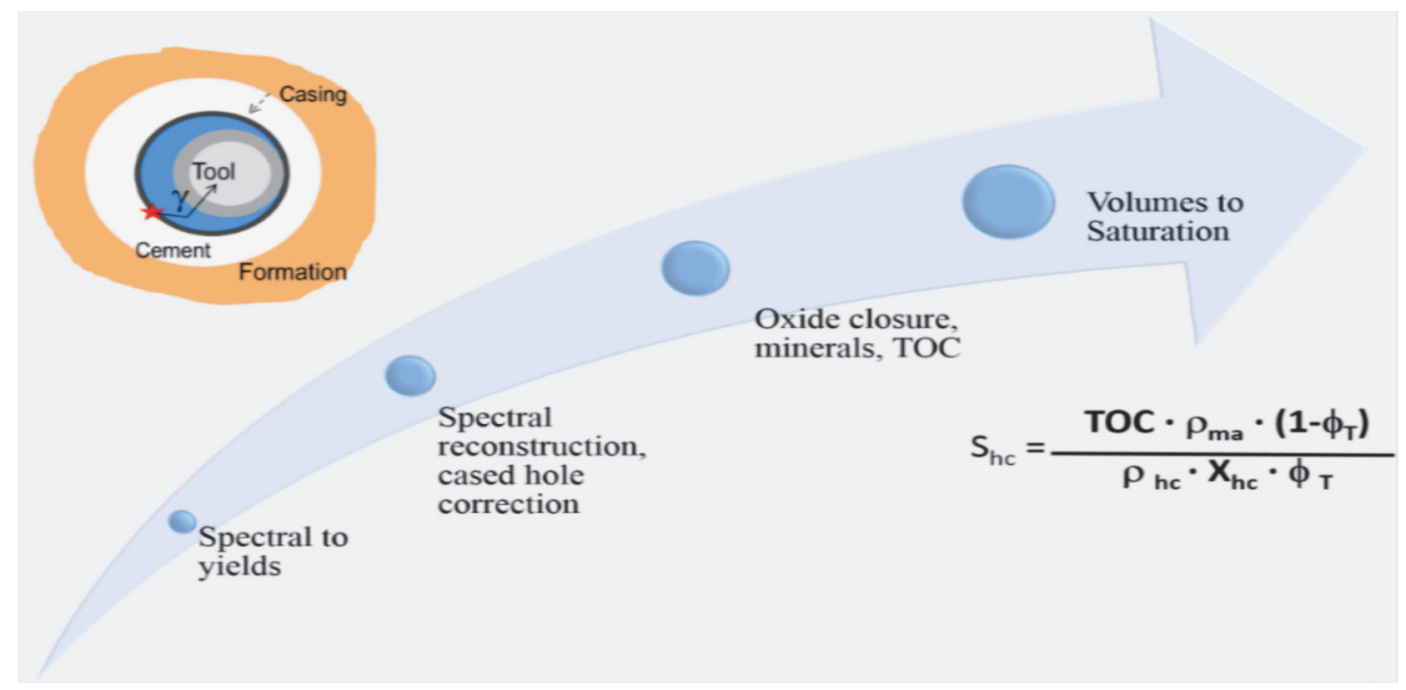

Figure 2. High Definition spectroscopy interpretation workflow - main steps from spectra to quantitative mineralogy, matrix properties, rock quality and fluid content 
The methodology begins with the acquisition at every depth of independent inelastic and total gamma ray capture spectra. A weighted linear regression of the combination of elements was performed for each set of data to output the Inelastic and Capture elemental yields.

Those are immediately converted to dry weight elements using a closure model, which combined input of both yields for more robust dry weight results. Simultaneously, the inorganic carbon dry weight is calculated from dry weight of dolomite, calcite, and other calcium minerals, and is subtracted from Total Carbon to obtain Organic Carbon dry weight. The dry weight of the main elements which are measured and selected using the innovative closure model for the case study are described in the Figure 3.

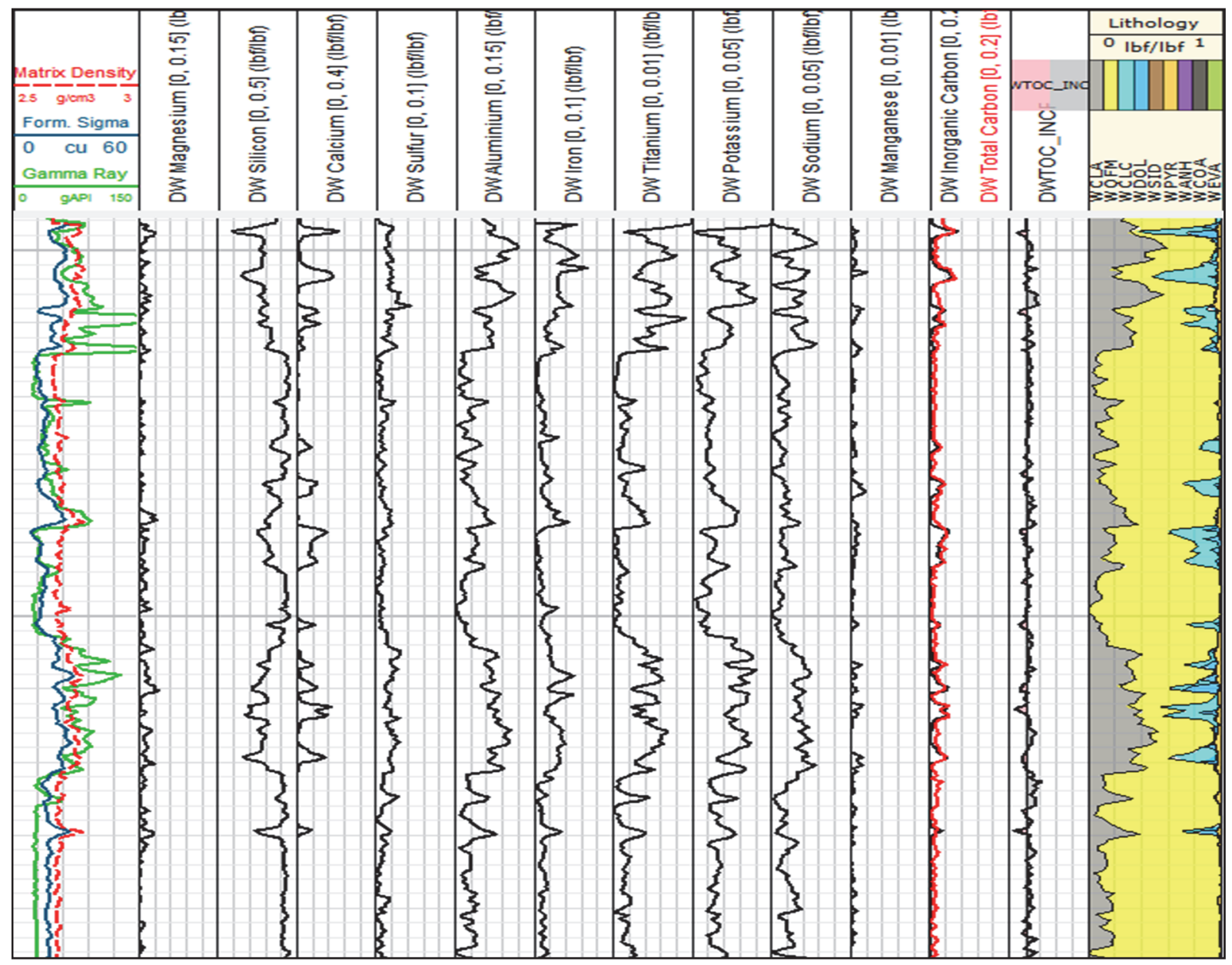

Figure 3. Stand-alone spectroscopy interpretation displaying dry weight elements and main lithology outputs $(1: 500$ scale $)$

The TOC expressed as weight fraction of organic carbon per unit mass of matrix component is converted into a volume fraction of hydrocarbon by integrating the total porosity from neutron-density corrected for matrix properties using the matrix density from spectroscopy itself. The computation uses the following equation (1). Note this equation is shown in Figure 2

$$
\mathrm{S}_{\mathrm{hc}}=\frac{\mathrm{TOC} \cdot \rho_{\mathrm{ma}} \cdot\left(1-\phi_{\mathrm{T}}\right)}{\rho_{\mathrm{hc}} \cdot \mathrm{X}_{\mathrm{hc}} \cdot \phi_{\mathrm{T}}}
$$

where

TOC is the Total Organic Carbon weight fraction from the spectroscopy log $\rho_{\text {ma }}$ is the matrix density from the spectroscopy log 
$\phi_{\mathrm{T}}$ is the Total Porosity using Neutron-Density logs with matrix property from the spectroscopy log

$\mathrm{X}_{\mathrm{hc}}, \rho_{\mathrm{hc}}$ are the carbon fraction and density of the formation oil, respectively in the bottom part of the logged interval, where the neutron-density data are not available, we were able to compute quantitative fluid saturation using the same methodology, but assuming a fixed total porosity.

\section{Results}

The advanced elemental spectroscopy measurement has been used in the field in cased-hole conditions to confirm the formation fluids and producibility of the reservoir intervals, where wellbore instability issues prevented openhole logs acquisition.

In this formation type, as indicated by the elemental composition and mineralogy assembly interpreted by the tool, any measured TOC could be directly attributed to the presence of hydrocarbon. Its calculation was done independently, without the risk of bias introduced by conventional models and the time delay caused by waiting for laboratory sample analysis are eliminated. Hydrocarbon saturation is directly inferred from TOC and porosity logs from cased-hole neutron-density measurements, which also proved valuable in achievivng a conclusive evaluation.The data analysis and interpretation of formation fluids was performed on time to enable immediate decision making and avoiding the risk of unwanted water production.

Figure 4 summarizes the results of the volumetric interpretation of mineralogy and fluids. Based on field knowledge the selected oil density for the spectroscopy based oil saturation is $0.927 \mathrm{~g} / \mathrm{cc}$, with carbon fraction about 0.85 .

The log results confirmed the presence of quality sands intercalated by shale layers and cementation at certain levels. The accurate mineralogy helps in the understanding of other logs response and provides confident fluid volumes and saturation. Most of the reservoirs described by the spectroscopy measurement have low carbon content, indicating the well would produce water; a critical information for proper decision making.

\section{Discussion and Conclusion}

This advanced technology combines a very high-resolution scintillator and a high-output pulsed neutron generator to measure an expanded set of elements which, in turn, increases the ability to properly interpret complex formations and perform fluid typing in a powerful predictive petrophysics workflow.

The case study provides a benchmark for detailed applications of the spectroscopy measurement to other development fields where formation evaluation is complex and the logging program is limited by operational or budget constraints.

Main rock properties and fluid saturation were accurately measured as presented in Figure 4. Additionally, the elemental concentrations and computed mineralogy from the advanced spectroscopy measurement were important to explain and validate the response of conventional logs and ability to use them in a combined interpretation.

The calculation of oil volume was done independently and immediately after logging the service, without the need of local calibration or the time delay while waiting for laboratory sample analysis. The robust saturation measurement was achieved regardless of formation water resistivity, saturation equation parameters, or clays effect, solving for the uncertainty related to the fluid content and potential for oil bearing layers. The result of the analysis changed the way the operator was looking at their development plans and requirement to extend the assessment of the field structure and reservoirs' connectivity.

The comprehensive data analysis confirm that the organic carbon log derived from geochemical spectroscopy logs can be used to obtain reliable estimates of hydrocarbon saturation in a wide range of environmental conditions and formations, including acquisition behind casing with previously inaccurate or unknown hole and formation properties. 


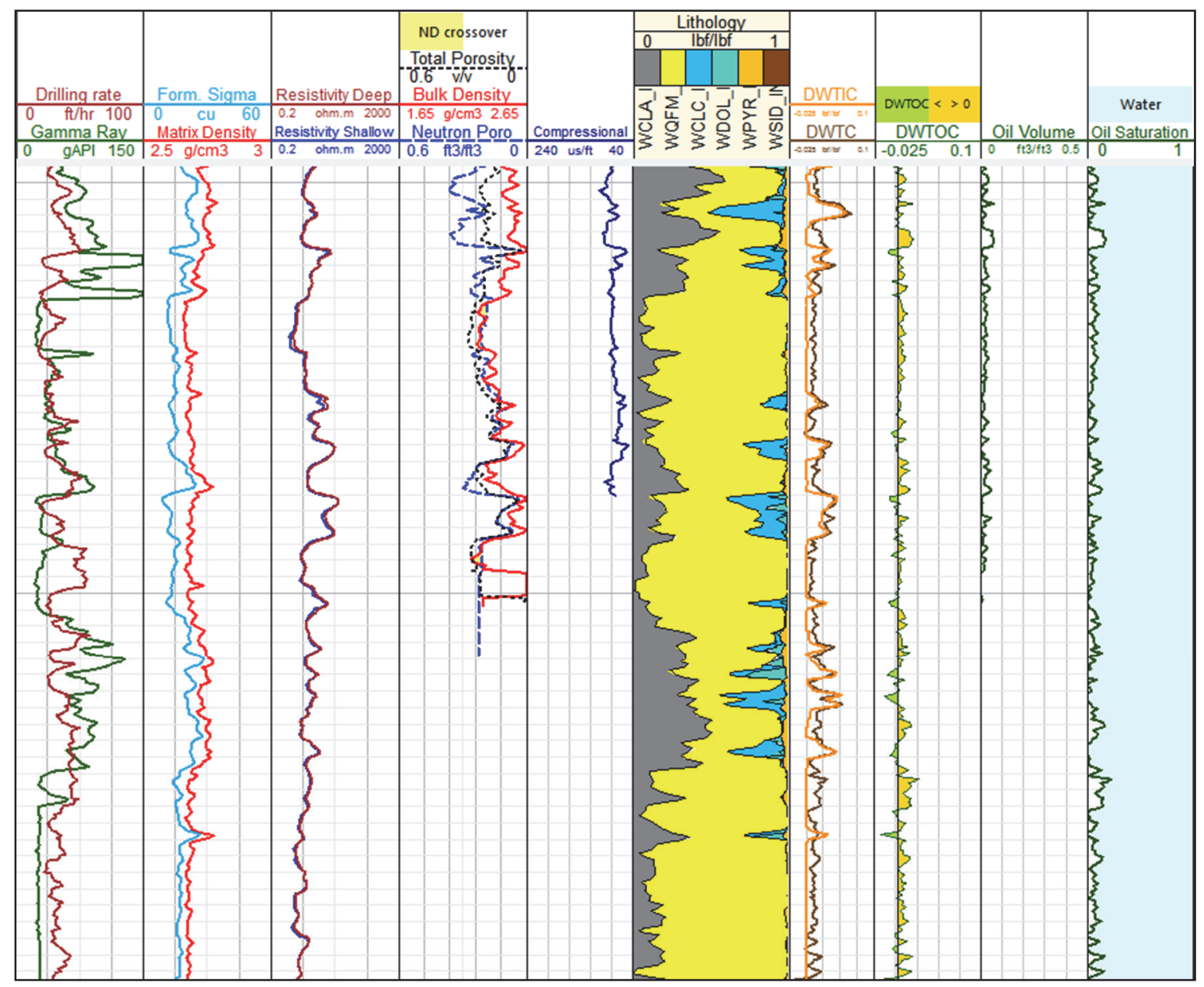

Figure 4. Composite log plot display with high definition spectroscopy data integrated to conventional logs outputs. Quantitative mineralogy, clay content, matrix density as well as accurate carbon content are provided by the measurement in stand-alone mode and adding unique value to rock quality and fluid content validation.

$(1: 500$ scale $)$

\section{Acknowledgments}

The authors are grateful to South Atlantic Petroleum (SAPETRO) management for their valuable sponsorship, collaboration and permission to publish this work.

\section{References}

Craddock, P. R. et al. (2013). Hydrocarbon Saturation from Total Organic Carbon Logs Derived from Inelastic and Capture Nuclear Spectroscopy, SPE 166297.

Herron, S. et al. (October 2014). Application and Quality Control of Core Data for the Development and Validation of Elemental Spectroscopy Log Interpretation. Petrophysics, 55(5), 392-414.

MacGregor, D. S., Robinson, J., \& Spear, G. (2003). Play fairways of the Gulf of Guinea transform margin, The Geological Society of London.

Radtke, R. J. et al. (June 2012). A New Capture and Inelastic Spectroscopy Tool Takes Geochemical Logging to the Next Level, SPWLA 53rd Annual Logging Symposium.

\section{Copyrights}

Copyright for this article is retained by the author(s), with first publication rights granted to the journal.

This is an open-access article distributed under the terms and conditions of the Creative Commons Attribution license (http://creativecommons.org/licenses/by/4.0/). 